\title{
Integrin Alpha L as an Immune-Related Biomarker Correlated with the Prognosis and Effect of Immunotherapy in Gliomas
}

\section{Jinyang Ma}

Department of neurosurgery, China Three Gorges University\&Yichang Central People's Hospital

\section{Lei Wang}

Department of neurosurgery, China Three Gorges University\&Yichang Central People's Hospital

\section{Youdong Zhou}

Institute of Neurology, China Three Gorges University

\section{Changtao Fu}

Department of neurosurgery, China Three Gorges University\&Yichang Central People's Hospital

\section{Song Huang}

Department of neurosurgery, China Three Gorges University\&Yichang Central People's Hospital

Bojuan Lang ( $\square$ lbjuan2021@163.com)

Department of Pathology, China Three Gorges University\&Yichang Central People's Hospital

\section{Research Article}

Keywords: ITGAL, Glioma, Biomarker, Tumor immunity, Drug target prediction

Posted Date: January 10th, 2022

DOl: https://doi.org/10.21203/rs.3.rs-1193120/v1

License: @ (i) This work is licensed under a Creative Commons Attribution 4.0 International License. Read Full License 


\section{Abstract \\ Backgroud:}

Discovering effective immune-related biomarkers is vital to ensure efficient immunotherapy for glioma patients. Integrin Alpha L(ITGAL), essential to inflammatory and immune responses, have not been studied in gliomas, systematically.

\section{Methods}

RNA-seq data and corresponding clinical information of glioma patients were collected from The Cancer Genome Atlas (TCGA) and Chinese Glioma Genome Atlas (CGGA), and mRNA data of normal brain tissues were obtained in Genotype-Tissue Expression (GTEx) project. Wilcoxon test was performed to analyze the correlation of ITGAL expression and glioma subtypes. Univariate and multivariate cox proportional hazards regression, receiver operating characteristic (ROC) curves and Kaplan-Meier plots were used to evaluate the prognostic value of ITGAL in glioma. Functional enrichment analyses and immune infiltration analysis were performed to investigate the potential function in mediating the immune response in the tumor microenvironment. Finally, we evaluated the ability of ITGAL for predicting the efficacy of ICB treatment for patients.

\section{Results}

We found the up-regulation of ITGAL may predict a poor prognosis for glioma patients, the expression level increased with the increasing of WHO grade and 1p19q co-deletion status and IDH mutation status. The total methylation level and copy number variation of ITGAL were moderately correlated with its mRNA expression in LGG samples $(P<0.05)$. Furthermore, ITGAL was correlated with the immunosuppressive tumor microenvironment for the strong correlation with M2 macrophages and Tregs. Finally, GSEA showed the upregulation of ITGAL was mainly involved in the signal recognition and regulation between immune cells, and Toll-like receptor signaling pathway.

\section{Conclusion}

ITGAL is a novel tumor-related and immune-associated biomarker, which could predict the prognosis and effect of ICB therapy for glioma patients.

\section{Introduction}

Glioma is the most common primary tumors in the central nervous system with a 5-year survival rate less than $10 \%(1)$, the combination of surgical treatment with radiotherapy or chemotherapy is still the principal treatment of malignant gliomas for now(2). In recent years, the development of novel immunotherapies for different components of tumor immune microenvironment provides great prospects for adjuvant therapy of glioma. The immune system play an important role in immune surveillance, since the immune cells of the adaptive and innate immune system infiltrate the tumor microenvironment (TME) and participate in the regulation of tumor 
progression(3). However, the tumor inhibitory immune microenvironment gradually formed during the growth of glioma which limits the efficacy of immunotherapy to a certain extent(4). The use of checkpoint (PD-1,PDL-1 or CTLA-4) inhibitors in multiple solid tumors has achieved significant clinical success $(5,6)$, which has aroused interest in immune targeting strategies for glioma therapy. Accumulating evidence indicates that the immune checkpoint mechanism plays an important role in suppressing tumor-specific immune responses within the tumor microenvironment(7). Thus, predicting and monitoring the response of patients to treatment has become an urgent requirement for clinical development of immunotherapy. The level of immune checkpoints and tumor mutation burdens (TMB) could be indicators evaluating the effect respond to immune checkpoint blockade (ICB) treatment $(8,9)$. TMB was considered as a quantitative measure of the total number of nonsynonymous mutations per coding area of the tumor genome, a surrogate marker of tumor immunogenicity reflecting neoantigen load(10) and an important indicator for successful immunotherapy(11). Besides, a computational method, Tumor Immune Dysfunction and Exclusion (TIDE), was created to determine the characteristics of T cell dysfunction in solid tumor tissues by detecting the expression pattern of each genes in tumor interacting with the level of cytotoxic T lymphocytes (CLT) invasion to affect the survival of patients(12). Now, the availability of biomarkers has greatly promoted the practice of oncology and began to be the basis of adjuvant therapy for glioma. However, the appropriate biomarkers for immunotherapy of glioma remain indeterminate. It is vital to further exploring potentially available immune-related biomarkers for predicting the prognosis and the effect of ICB therapy for cancers.

Integrin Alpha $L(I T G A L)$ belongs to the integrin a chain family, which encodes integrin a $L$ chain, which mainly contacts with $\mathrm{MHC}$ molecules on antigen presenting cells through $\mathrm{T}$ cell receptor, thus plays a role in $\mathrm{T}$ cell activation(13). Emerging evidence suggests that integrin also play an important role in angiogenesis and cancer development, including cell migration, apoptosis and proliferation(14-16). Related research found that ITGAL may be associated with Tumor Infiltrating Lymphocytes (TILs) and independent predictors of early prognosis in ovarian cancer(17), it was also reported to be a potential diagnostic biomarker for interstitial cystitis/bladder pain syndrome(18). ITGAL is essential for inflammation and immune response, regulating the adhesion and costimulation interaction between CD4 (+ T cells) and other cells, It was found that demethylation of ITGAL regulatory elements and subsequent overexpression in CD4 (+) T cells may mediate immunological abnormalities and fibrosis of systemic sclerosis(19).However, whether ITGAL was a novel immune prognostic biomarker associated with glioma may need to be further investigated.

Here, we conjectured that the interaction between ITGAL and the immune status has a certain significance for the diagnosis and prognosis of glioma. In addition, the correlation between ITGAL and clinical outcomes would be investigated. Moreover, based on the correlation between ITGAL and expression level of immune checkpoint, TMB and TIDE, the predictive value of ICB for patients with glioma will be explored. Finally, the components targeting ITGAL were analyzed.

\section{Materials And Methods}

\section{Publicly available database}

RNA-seq data and corresponding clinical information of glioma patients were collected from The Cancer Genome Atlas (TCGA) (http://cancergenome.nih.gov/), and the mRNA-seq data of normal brain tissues were 
downloaded from Genotype-Tissue Expression (GTEx) project, then a training set was established by emerging and then normalizing the data of TCGA and GTEx by R package "limma"(20)..Similarly, the RNA-seq and clinical information obtained from the datasets mRNAseq_693 and mRNAseq_325 in the Chinese Glioma Genome Atlas (CGGA) (http://www.cgga.org.cn) were mergerd and normalized as a validation set. All sample data with age less than 18 years old, survival time less than three months and incomplete information were removed. The training set included a total of 459 tumor and 1152 normal samples selected, The mRNAseq_693 and mRNAseq_325 dataset in CGGA are merged into one cohort as a validation set included a total of 681 samples selected.

\section{Patient samples}

The Institutional Ethics Committee approved this study of the Faculty of Medicine at Yichang Central People's Hospital. In total, 6 control samples from patients with cerebral hemorrhage, 28 samples from patients with lowgrade glioma, and 44 samples from patients with glioblastomas were collected during May 2019 and October 2021. No patients were treated with chemotherapy or radiotherapy before surgery.

\section{The expression level of ITGAL in tumor and normal tissue}

The expression levels of ITGAL mRNA in pan-cancer were investigated in TIMER 2.0 database (https://cistrome.shinyapps.io/timer/)(21), and the value of ITGAL in different grade gliomas and normal brain tissues were compared in training set and validation set by $\mathrm{R}$ package "limma", p-value less than 0.05 was used as a threshold for significance. To investigate the diagnosis role of ITGAL in glioma, the receiver operating characteristic (ROC) curve was built and the area under the ROC curve (AUC) was calculated by R packages "pROC".

\section{RNA extraction and quantitative real-time PCR}

The extraction of RNA of ITGAL from tissues and cells was carried out using TRIzol reagent (Invitrogen, Carlsbad, CA, USA). The PrimeScript RT Reagent Kit (RR047A, Takara, Japan) was used to synthesize cDNA. SYBR Premix Ex Taq II (RR820A, Takara, Kusatsu, Japan) and Bio-Rad CFX Manager 2.1 real-time PCR Systems (Bio-Rad, Hercules, CA, USA) were used to detect the ITGAL mRNA levels following the specifications provided by the manufacturers. The relative $\mathrm{Ct}$ method was used to compare the data of the experimental and control groups, and GAPDH was used as the internal control. The primer sequences of mRNA included the following: GAPDH 5'-GGAGCGAGATCCCTCCAAAAT-3' (Forward) and 5'-GGCTG TTGTCATACTTCTCATGG-3' (Reverse); ITGAL 5'- TGCTTATCATCATCACGGATGG-3' (Forward) and 5'- CTCTCCTTGGTCTGAAAATGCT-3' (Reverse).

\section{Investigate the prognostic value of ITGAL in glioma}

According to the median expression level of ITGAL in training and validation set respectively, the tumor samples were divided into high and low expression groups to investigate whether there were significant differences between two groups for overall survival (OS) of glioma patients by performing Kaplan-Meier (K-M) survival curve through R package "survival". ROC curve graph was used to evaluate the performance of prognostic predictive ability for 1-, 3- and 5-year OS. And K-M curve for disease free survival (DFS) in glioma was obtained from Gene Expression Profiling Interactive Analysis database (GEPIA, http://gepia.cancer-pku.cn/). Then Univariate and multifactorial Cox proportional hazards regression analysis was performed using R package "survival" to explore the independent risk factors in training and validation sets, factors with P-value $<0.05$ in 
univariate Cox regression analysis was selected in multifactorial Cox analysis and P-value two-tailed below 0.05 was considered statistically significant.

\section{Correlation of ITGAL with clinicopathological characteristics}

The relationship between the expression value of ITGAL and clinical subtypes was investigated by Wilcoxon test in TCGA and CGGA. ( $p<0.05$ was considered significant). Here, the 459 samples in TCGA and 681 samples in CGGA were apart divided into two groups by World Health Organization (WHO) grade, age (cutline was 45 years old)(22), IDH mutation status, 1p19q-codeletion status, gender and etc., the levels of ITGAL in different groups were showed in boxplots plotting by R package "ggpubr".

\section{DNA amplification and hypomethylation analysis}

To further explore the potential mechanism of ITGAL regulation in gliomas, its genetic and epigenetic alterations were explored in TCGA-LGG and TCGA-GBM cohorts according to the cBioPortal database

(http://www.cbioportal.org/). According to the tumor samples with mRNA data, copy number variation (CNV) data, and DNA methylation data. Linear regression analyses were performed among mRNA expression level of ITGAL and corresponding data of CNV and methylation. A p-value less than 0.05 was considered significant.

\section{The protein-protein interaction networks(PPI)}

The PPI of ITGAL were predicted by STRING(https://string-db.org/), the correlation coefficient greater than 0.99 and P-value below 0.001 were considered statistically significant. Then the PPI network was rebuilt through the software Cytoscape(23).

\section{tumor-infiltrating immune cells and immune infiltration level profiles}

The stromal score, immune score and tumor purity of the tumor samples in TCGA-glioma were calculated by Rpackage "estimate", according to the median value of ITGAL, tumor samples were divided into high and low expression groups, then the immune scores above in two groups were compared, $\mathrm{K}-\mathrm{M}$ survival analysis and logrank test ( $p$-value less than 0.05 means the results are statistically significant) were performed to investigate the prognostic value of immune scores. The abundance of tumor infiltrating immune cells (TIICs) in glioma tissues were calculated by R-package "cibersort", and the abundance of TIICs in low and high ITGAL expression group were compared using Wilcoxon test, immune cells with $\mathrm{P}$ value $<0.05$ was considered significantly different in two groups. The correlation between TIICs and ITGAL expression level was further investigated by Spearman test.

\section{Assess the value of ITGAL for predicting immunotherapy response}

Spearman's correlation analysis was used to evaluate the relationship between ITGAL and immune checkpoints. In the high and low ITGAL expression group, the value of TMB and TIDE were compared using R package "limma".

\section{Gene set enrichment analysis(GSEA)}


GSEA was performed to evaluate the correlations between ITGAL expression (high vs. low) and cancer-related pathways using the gene sets (c5.all.v5.1) downloaded from Molecular Signatures Database (MSigDB). The gene sets with nominal p-value 0.05 and FDR $<0.25$ were considered significantly enriched.

\section{Predict targets of ingredients in traditional Chinese medicine (TCM)}

Network pharmacological analysis was performed by predicting targets of ingredients in TCM through Traditional Chinese Medicine Systems Pharmacology Database and Analysis Platform (TCMSP, http://tcmspw.com/tcmsp.php). The molecular docking studies among effective ingredient and ITGAL were performed using Autodock Vina 1.1.2.

\section{Results}

\section{Up-regulation of ITGAL expression level in gliomas}

Compared to the normal tissue, pan-cancer analysis showed the ITGAL expression level is lower in Bladder Urothelial Carcinoma, Cholangiocarcinoma and so on, while higher in Breast invasive carcinoma, Kidney renal clear cell carcinoma and Stomach adenocarcinoma (Figure 1A). The ITGAL expression is significantly upregulated in glioma tissues in training set (Fig.1B), and the level of ITGAL mRNA was validated in our hospital samples (Fig. 1C), which means ITGAL may be involved in the progression of glioma. The AUC of ROC curve for diagnosing glioma using the level of ITGAL was 0.758 in training set (Fig. 1D).

\section{The prognostic value of ITGAL in gliomas}

Kaplan-Meier survival analysis performed to investigate the correlation between the gene with clinical prognosis indicated that high expression level of ITGAL significantly associated with the poor OS and DFS of patients. In TCGA cohort, as shown in the figure $2 \mathrm{~A}-\mathrm{C}$, the patients in low expression group had better OS than in the high expression group including in the all WHO grade gliomas and lower grade gliomas (LGG, WHO II and WHO III), while in the glioblastoma (GBM) group there was no correlation between ITGAL and OS considering that the number of patients with GBM was too small and the survival time of patients was too short. The AUC of ROC curve for predicting 1-, 3- and 5-year OS were respectively $0.709,0.631$ and 0.622 (Fig. 2D). In CGGA cohort, higher expression of ITGAL was significantly associated with poor survival as the Figure 2E-G showed and the AUC of ROC curve for predicting 1-, 3- and 5-year OS in CGGA were respectively $0.614,0.630$ and 0.660 (Fig. 2H). Figure 2I-K showed that patients in high expression group had poorer DFS than in the low expression group according to GEPIA database. In Tab.1, the univariate cox logistic regression analysis and multivariate cox logistic regression analysis of OS indicated that grade, IDH. status, age and ITGAL expression could be independent factors to predict the prognosis of glioma patient. While in CGGA cohort, as the Tab. 2 shown, 1 19q co-deletion could also be an additional independent factor. These results demonstrated that the upregulation of ITGAL indicated a poorer prognosis of glioma patients.

\section{ITGAL expression was significantly correlated with clinicopathological characteristics}

The basic characteristics of the patients from TCGA and CGGA were sorted out in the Tab.3. As the Figure 3 showed, in TCGA, the level of ITGAL increased with increasing WHO grade(II-IV) of glioma and correlated with 
the clinical subtypes, including age, 1p19q.-codeletion status and IDH mutation status. while in CGGA, the value of ITGAL were no significant difference between Lower WHO grade (WHO II vs. WHO III) and age groups. No specificity of expression was observed in different genders in both TCGA and CGGA.

\section{Mutation and DNA hypomethylation contributed to ITGAL expression regulation in glioma}

To further explore the mechanism of ITGAL dysregulation in gliomas, we analyzed its genetic and epigenetic alterations in TCGA-LGG and GBM cohorts. Figure 4A showed a landscape of ITGAL's common mutation sites and mutation types. In addition, LGG samples with mRNA data and copy number variation (CNV) data, shallow deletion was significantly associated with upregulation of ITGAL mRNA (Anova $p<0.05$ ) (Figure 4B). Moreover, linear regression analyses indicated that the total methylation level of ITGAL was moderately and negatively correlated with the mRNA expression in LGG samples (Figure 4C, Spearman's $r=-0.55$, Pearson's $r=-0.47, P<$ 0.001). However, there was no significant correlation between the mRNA expression of ITGAL and the level of CNV and methylation in GBM samples (Figure 4D, E).

\section{The association between ITGAL and tumor immune microenvironment in glioma}

The relationship between ITGAL and tumor immune microenvironment was investigated in terms of the level of immune infiltration and the abundance of immune cells. As the Fig. 5A-C showed, the immune infiltration level including immune score and stromal score were higher in the high expression group of ITGAL, while tumor purity was significantly higher in the low expression group. Then the prognostic significance of infiltration level of immune cells was identified by K-M curve (Fig. 5D-F). In the high and low expression group of ITGAL, the TIICs with high level were macrophages, monocytes, mast cells and memory resting CD 4 T cells, and the difference analysis of immune infiltrating cells showed that the level of M2 macrophages and T regulatory cells (Tregs) of high ITAGL expression group were significantly higher than those of low expression group while the active Mast cells were in the opposite results (Fig. 5G). Further correlation analysis showed in the figure $5 \mathrm{H}-\mathrm{J}$ that the number of M2 macrophages and Tregs were positively correlated to the level of ITGAL, the values of rho were 0.2 and 0.18 respectively, both $p<0.01$, while the abundance of actived mast cells was negatively associated with the expression of ITGAL, rho $=-0.33, p<0.001$.

\section{Patients with higher ITGAL expression level may benefit more from ICB treatment}

Ten co-expressed genes in PPI network were shown in Fig. 6A, ITGB1, ITGB2, ITGB3, ITGB7, CD28, CTLA4, ICAM1, ICAM2ICAM3 and ICAM5. Spearman correlation test (Fig. 6B-D) performed suggests that the expression level of ITGAL was significantly positively correlated with main immune checkpoints including CTLA4 (rho: 0.48, $p<0.001$ ), PD-1, rho: 0.56, $p<0.001$ ) and PDL-1 (rho: 0.52, $p<0.001$ ). In this study, TMB was higher in the high ITGAL expression group than in the low expression group (Figure 6E, $p<0.001$ ), TIDE score calculated was significantly higher in the low expression group (Figure 6F, $p<0.001$ ). These results indicated that ITGAL may be a potential indicator evaluate the efficacy of ICB therapy and patients with higher ITGAL expression level may benefit more from immunotherapy.

\section{GSEA analysis}

GSEA performed to investigate the potential pathways and process which ITGAL involved in. The results suggested that the most enriched terms positively related to ITGAL were 
“GO_REGULATION_OF_MAST_CELL_ACTIVATION_INVOLVED_IN_IMMUNE_RESPONSE”,

“GO_PATTERN_RECOGNITION_RECEPTOR_SIGNALING_PATHWAY”, “HP_LEUKOCYTOSIS” and

"GO_TOLL_LIKE_RECEPTOR_SIGNALING_PATHWAY", and the top four enriched gene sets negatively related to ITGAL were “GO_NEURON_CELL_CELL_ADHESION”, GO_GLUTAMATE_SECRETION”,

“HP_EPILEPTIC_ENCEPHALOPATHY” and “GO_GABA_ERGIC_SYNAPSE”. The nominal p-value and normalized enrichment score were shown in figure 7.

\section{Ingredient in TCM targeting ITGAL}

The effective ingredient of Bombyx Mori L, Momordicae Fructus, Ecliptae Herba and Equiseti Hiemalis Herba, nicotine, targeting ITGAL was obtained. Secondary structure of ITGAL and 3D structure of nicotine were shown in Fig. 8A and Fig. 8B respectively. The result of the molecular docking was viewed from different angles (Fig. $8 \mathrm{C}-\mathrm{E})$. These predicted docking sites could facilitate further studies of ITGAL-targeting drugs and related mechanisms

\section{Discussion}

Glioma leading to high mortality is the most common type of brain tumor in human, importantly, bio-markers of tumor could play vital roles for diagnosis, prognosis and targeting therapy in cancer(24). Advances in genetics and molecular biology have recently enabled the rapid development of personalized cancer management. No previous studies have reported the function of ITGAL in solid tumors, we investigated the expression level of ITGAL in normal and tumor tissues and evaluated the diagnostic and prognostic value of ITGAL for glioma by K-M survival analysis, ROC curve and multivariate cox regression analysis. In addition, ITGAL was found significantly associated with TIME in glioma, which are correlated to worse OS. These indicated that ITGAL may be a novel immune-related biomarker in glioma. Moreover, the expression of ITGAL was positively associated with WHO grade of patients, which indicated that ITGAL may be a tumor promoting gene in glioma. In this research, the upregulation of ITGAL expression may appear in the subtypes such as non-co-deletion of $1 p 19 q$ and IDH1 wild type. Generally, in low-grade gliomas and IDH-mutant glioblastomas, mutations in IDH predict significantly improved OS compared to wild-type IDH gliomas(25) (26), Co-deletion of 1p19q was first described in 1994 and while its exact biologic effect in gliomas is not clear, its presence has been associated with increased chemosensitivity and hence a more favorable prognosis $(27,28)$. It suggested that ITGAL may be involved in the development of tumor related molecular subtypes, and upregulation of its expression may be a trigger inducing IDH into wild type state or 1 p19q into non-co-deletion status so as to enhance the resistance to adjuvant therapy (chemotherapy or immunotherapy) and then affect the prognosis of patients.

In this study, ITGAL was mainly involved in the signal recognition and regulation between immune cells in glioma, participating Toll-like receptor signaling pathway and the production of tumor necrosis factor superfamily cytokines according to the results of GSAE. Toll like receptor (TLR) is a single transmembrane non catalytic protein expressed on the surface of transmembrane cells, such as macrophages and dendritic cells, they play an important role in innate immune response and antigen-specific acquired immunity(29). TLR pathway may play a key role in some tumorigenesis and development. Therefore, some genetic variations in this pathway may be used as potential biomarkers for clinical outcomes of some solid tumors such as lung cancer(30, 31). 
According to their different functions, Tumor-associated macrophages (TAMs) are divided into two main types: M1 type was activated by classical pathway mainly promoting the aggregation of inflammatory factors against tumorigenesis, progression and metastasis, while $\mathrm{M} 2$ type activated by bypass pathway, playing the opposite role(32). TAMs play an important role in promoting glioma progression(33,34), so that treatment targeting TAMs has become an important strategy in the adjuvant treatment of glioma. In addition, Tregs could also suppress the function of immune effector cells through a variety of mechanisms and were considered as key factors in tumor immune escape in most cancers(35), which represent a critical barrier to immunotherapy of tumors, targeting Tregs may also be a key point in immunotherapy for immune precision medicine. Our study found that the expression of ITGAL was significantly correlated with M2 macrophages and Tregs, which both indicated that ITGAL may be a tumor-related immunosuppressive gene, targeting ITGAL may provide a novel and effective strategy for relieving the state of immunosuppression and activating the killing effect of tumor infiltrating immune cells in TME.

Immunotherapy is increasingly acknowledged as an effective treatment for several canine cancers(36). However, due to the presence of the blood-brain barrier, ICB is largely restricted in central nervous system (CNS) tumor since the circulating immune cells were difficult to reach the CNS(37). In this study, relating the PPI network of ITGAL, ten nodes with strong co-expression relationship were screened, which were reported to be tumor associated integrin family members(17) and intercellular adhesion molecules(38, 39). Adhesion molecules and integrin were considered to play an important role in helping lymphocytes at the blood-brain barrier could primarily promotes homing of T cells to central nervous system to play the role of tumor killing(40-42). ITGAL as a member of integrin-family cell adhesion molecule may induce the activated $T$ cells to homing into the CNS and exert the tumor killing effect in the process of ICB treatment. Therefore, the upregulation of ITGAL may provide a possibility for immunotherapy of glioma. Although promising, the therapeutic efficacy of immune therapy, such as ICB, may still be limited $(43,44)$. The efficacy of ICB also depends on the expression of immune checkpoint. Meanwhile, the greater the mutation load of tumor, the easier the tumor is recognized by the immune system and killed by CD $4 / 8 \mathrm{~T}$ cells $(8,45)$. On the other hand, most cancer ICB require T cells to perform anti-neoplastic effects(46), TIDE algorithm predicted the effect of ICB by focusing on the function activation and aggregation of T cells in tumor and TIDE score was considered as an accurate predictor of response to ICB(47). Due to the significant correlation between ITGAL and indicators of ICB including the expression level of immune checkpoints, TMB and TIDE score, ITGAL may be a novel indicator to predict the benifit of ICB treatment and patients with higher ITGAL expression level may benefit more from immunotherapy.

In addition, nicotine was reported to act primarily by activation of nicotine acetylcholine receptors (nAChRs) and binds to these receptors with a higher affinity than acetylcholine(48). Several research indicated that nicotine may contribute to the development of cancers by increasing the proliferative potential and anti-apoptotic ability of cells $(49,50)$. In our study, nicotine could specifically bind to ITGAL protein in a relatively strong way, which provides some enlightenment. The potential mechanisms of nicotine targeting ITGAL protein and activating downstream cancer-related signaling pathways need to be further studied.

\section{Conclusion}


In conclusion, ITGAL is an immune-related and cancer-promoting gene, which could specifically diagnose and accurately predict the prognosis of patients in gliomas. ITGAL was involved in immunosuppressive TME, patients with higher expression level of ITGAL may be more sensitive to ICB therapy. Moreover, this study not only identified a novel immune biomarker, but also provided a new perspective to understand the underlying relationship between tumor immune microenvironment and immunotherapy in gliomas.

\section{Declarations}

\section{Ethics approval and consent to participate}

Institutional Ethics Committee of the Faculty of Medicine at Yichang Central People's Hospital approval (YCZX2021-T112) was obtained to carry out the study within its facilities. A written informed consent was obtained from both parents of the participant. All methods were carried out in accordance with relevant guidelines and regulations.

\section{Consent for publication}

Not applicable.

\section{Availability of data and materials}

Publicly available datasets were analysed in this study. This data can be found below:

1. TCGA, https://www.cancer.gov/;

2. GEPIA, http://gepia.cancer-pku.cn/detail.php;

3. GTEx, https://www.gtexportal.org/home;

4. TIMER, https://cistrome.shinyapps.io/timer/

5. STRING, https://string-db.org/cgi/input.pl

\section{Competing interest}

The authors declare that they have no conflict of interest.

\section{Funding}

Not applicable.

\section{Authors' contributions}

JM and BL contributed to conception and design of this study. LW, YZ, CF, and SH contributed to the analysis and interpretation of data. All authors read and approved the final manuscript.

\section{Acknowledgments}

We gratefully acknowledge The Cancer Genome Atlas pilot project which made the genomic data and clinical data of glioma available. 


\section{References}

1. Ostrom QT, Cioffi G, Gittleman H, Patil N, Waite K, Kruchko C, Barnholtz-Sloan JS. CBTRUS Statistical Report: Primary Brain and Other Central Nervous System Tumors Diagnosed in the United States in 20122016. Neuro-oncology (2019) 21:v1-v100.

2. Wang Q, Shen M, Sun Y, Zhang X, Huang C, Chen J, Li R, Duan Y. Thermoresponsive nanocomposite gel for local drug delivery to suppress the growth of glioma by inducing autophagy. Autophagy (2017) 13:11761190. doi: 10.1080/15548627.2017.1320634

3. Matsushita H, Vesely MD, Koboldt DC, Rickert CG, Uppaluri R, Magrini VJ, Arthur CD, White JM, Chen Y-S, Shea LK, et al. Cancer exome analysis reveals a T-cell-dependent mechanism of cancer immunoediting. Nature (2012) 482: doi: 10.1038/nature10755

4. Garner $\mathrm{H}$, de Visser KE. Immune crosstalk in cancer progression and metastatic spread: a complex conversation. Nature reviews Immunology (2020) 20: doi: 10.1038/s41577-019-0271-z

5. Emens LA, Ascierto PA, Darcy PK, Demaria S, Eggermont AMM, Redmond WL, Seliger B, Marincola FM. Cancer immunotherapy: Opportunities and challenges in the rapidly evolving clinical landscape. European journal of cancer (Oxford, England: 1990) (2017) 81: doi: 10.1016/j.ejca.2017.01.035

6. Lipson EJ, Forde PM, Hammers H-J, Emens LA, Taube JM, Topalian SL. Antagonists of PD-1 and PD-L1 in Cancer Treatment. Seminars in oncology (2015) 42: doi: 10.1053/j.seminoncol.2015.05.013

7. Huang C-Y, Chiang S-F, Ke T-W, Chen T-W, You Y-S, Chen WT-L, Chao KSC. Clinical significance of programmed death 1 ligand-1 (CD274/PD-L1) and intra-tumoral CD8+ T-cell infiltration in stage II-III colorectal cancer. Scientific reports (2018) 8: doi: 10.1038/s41598-018-33927-5

8. Wang L, Ge J, Lan Y, Shi Y, Luo Y, Tan Y, Liang M, Deng S, Zhang X, Wang W, et al. Tumor mutational burden is associated with poor outcomes in diffuse glioma. BMC cancer (2020) 20: doi: 10.1186/s12885-0206658-1

9. Furuse M, Kuwabara H, Ikeda N, Hattori Y, Ichikawa T, Kagawa N, Kikuta K, Tamai S, Nakada M, Wakabayashi T, et al. PD-L1 and PD-L2 expression in the tumor microenvironment including peritumoral tissue in primary central nervous system lymphoma. BMC cancer (2020) 20: doi: 10.1186/s12885-02006755-y

10. Tovoli F, De Lorenzo S, Trevisani F. Immunotherapy with Checkpoint Inhibitors for Hepatocellular Carcinoma: Where Are We Now? Vaccines (2020) 8: doi: 10.3390/vaccines8040578

11. Song W, Shen L, Wang Y, Liu Q, Goodwin TJ, Li J, Dorosheva O, Liu T, Liu R, Huang L. Synergistic and low adverse effect cancer immunotherapy by immunogenic chemotherapy and locally expressed PD-L1 trap. Nature communications (2018) 9: doi: 10.1038/s41467-018-04605-x

12. Jiang P, Gu S, Pan D, Fu J, Sahu A, Hu X, Li Z, Traugh N, Bu X, Li B, et al. Signatures of T cell dysfunction and exclusion predict cancer immunotherapy response. Nature medicine (2018) 24: doi: 10.1038/s41591018-0136-1

13. Blanco FJ, Ojeda-Fernandez L, Aristorena M, Gallardo-Vara E, Benguria A, Dopazo A, Langa C, Botella LM, Bernabeu C. Genome-wide transcriptional and functional analysis of endoglin isoforms in the human promonocytic cell line U937. Journal of cellular physiology (2015) 230: doi: 10.1002/jcp.24827 
14. Silva R, D’Amico G, Hodivala-Dilke KM, Reynolds LE. Integrins: the keys to unlocking angiogenesis. Arteriosclerosis, thrombosis, and vascular biology (2008) 28: doi: 10.1161/ATVBAHA.108.172015

15. Winograd-Katz SE, Fässler R, Geiger B, Legate KR. The integrin adhesome: from genes and proteins to human disease. Nature reviews Molecular cell biology (2014) 15: doi: 10.1038/nrm3769

16. Seguin L, Desgrosellier JS, Weis SM, Cheresh DA. Integrins and cancer: regulators of cancer stemness, metastasis, and drug resistance. Trends in cell biology (2015) 25: doi: 10.1016/j.tcb.2014.12.006

17. Wu A, Zhang S, Liu J, Huang Y, Deng W, Shu G, Yin G. Integrated Analysis of Prognostic and Immune Associated Integrin Family in Ovarian Cancer. Frontiers in genetics (2020) 11: doi:

10.3389/fgene.2020.00705

18. Saha SK, Jeon T-I, Jang SB, Kim SJ, Lim KM, Choi YJ, Kim HG, Kim A, Cho S-G. Bioinformatics Approach for Identifying Novel Biomarkers and Their Signaling Pathways Involved in Interstitial Cystitis/Bladder Pain Syndrome with Hunner Lesion. Journal of clinical medicine (2020) 9: doi: 10.3390/jcm9061935

19. Wang Y, Shu Y, Xiao Y, Wang Q, Kanekura T, Li Y, Wang J, Zhao M, Lu Q, Xiao R. Hypomethylation and overexpression of ITGAL (CD11a) in CD4(+) T cells in systemic sclerosis. Clinical epigenetics (2014) 6: doi: 10.1186/1868-7083-6-25

20. Yan K, Yang Y, Zhang Y, Zhao W, Liao L. Normalization Method Utilizing Endogenous Proteins for Quantitative Proteomics. Journal of the American Society for Mass Spectrometry (2020) 31: doi: 10.1021/jasms.0c00012

21. Li T, Fu J, Zeng Z, Cohen D, Li J, Chen Q, Li B, Liu XS. TIMER2.0 for analysis of tumor-infiltrating immune cells. Nucleic acids research (2020) 48: doi: 10.1093/nar/gkaa407

22. Hou H, Zhang C, Qi X, Zhou L, Liu D, Lv H, Li T, Sun D, Zhang X. Distinctive targetable genotypes of younger patients with lung adenocarcinoma: a cBioPortal for cancer genomics data base analysis. Cancer biology \& therapy (2020) 21: doi: 10.1080/15384047.2019.1665392

23. Shannon P, Markiel A, Ozier O, Baliga NS, Wang JT, Ramage D, Amin N, Schwikowski B, Ideker T. Cytoscape: a software environment for integrated models of biomolecular interaction networks. Genome research (2003) 13: doi: 10.1101/gr.1239303

24. Locascio JJ, Eberly S, Liao Z, Liu G, Hoesing AN, Duong K, Trisini-Lipsanopoulos A, Dhima K, Hung AY, Flaherty AW, et al. Association between a-synuclein blood transcripts and early, neuroimaging-supported Parkinson's disease. Brain: a journal of neurology (2015) 138: doi: 10.1093/brain/awv202

25. Karpel-Massler G, Ishida CT, Bianchetti E, Zhang Y, Shu C, Tsujiuchi T, Banu MA, Garcia F, Roth KA, Bruce JN, et al. Induction of synthetic lethality in IDH1-mutated gliomas through inhibition of Bcl-xL. Nature communications (2017) 8: doi: 10.1038/s41467-017-00984-9

26. Watanabe T, Nobusawa S, Kleihues P, Ohgaki H. IDH1 mutations are early events in the development of astrocytomas and oligodendrogliomas. The American journal of pathology (2009) 174: doi: 10.2353/ajpath.2009.080958

27. Reifenberger J, Reifenberger G, Liu L, James CD, Wechsler W, Collins VP. Molecular genetic analysis of oligodendroglial tumors shows preferential allelic deletions on $19 q$ and $1 p$. The American journal of pathology (1994) 145: http://www.ncbi.nlm.nih.gov/pubmed/7977648

28. Ang SYL, Lee L, See AAQ, Ang TY, Ang BT, King NKK. Incidence of biomarkers in high-grade gliomas and their impact on survival in a diverse SouthEast Asian cohort - a population-based study. BMC cancer (2020) 
20: doi: 10.1186/s12885-020-6536-x

29. Khan AA, Khan Z, Warnakulasuriya S. Cancer-associated toll-like receptor modulation and insinuation in infection susceptibility: association or coincidence? Annals of oncology: official journal of the European Society for Medical Oncology (2016) 27: doi: 10.1093/annonc/mdw053

30. Wei F, Yang F, Li J, Zheng Y, Yu W, Yang L, Ren X. Soluble Toll-like receptor 4 is a potential serum biomarker in non-small cell lung cancer. Oncotarget (2016) 7: doi: 10.18632/oncotarget.9496

31. Ke X, Wu M, Lou J, Zhang S, Huang P, Sun R, Huang L, Xie E, Wang F, Gu B. Activation of Toll-like receptors signaling in non-small cell lung cancer cell line induced by tumor-associated macrophages. Chinese journal of cancer research = Chung-kuo yen cheng yen chiu (2015) 27: doi: 10.3978/j.issn.1000-9604.2015.03.07

32. Zong CC. Single-cell RNA-seq study determines the ontogeny of macrophages in glioblastomas. Genome biology (2017) 18: doi: 10.1186/s13059-017-1375-z

33. Lei X, Chen X, Quan Y, Tao Y, Li J. Targeting CYP2J2 to Enhance the Anti-Glioma Efficacy of Cannabinoid Receptor 2 Stimulation by Inhibiting the Pro-Angiogenesis Function of M2 Microglia. Frontiers in oncology (2020) 10: doi: 10.3389/fonc.2020.574277

34. Guo X, Xue H, Shao Q, Wang J, Guo X, Chen X, Zhang J, Xu S, Li T, Zhang P, et al. Hypoxia promotes gliomaassociated macrophage infiltration via periostin and subsequent M2 polarization by upregulating TGF-beta and M-CSFR. Oncotarget (2016) 7: doi: 10.18632/oncotarget.11825

35. Li C, Jiang P, Wei S, Xu X, Wang J. Regulatory T cells in tumor microenvironment: new mechanisms, potential therapeutic strategies and future prospects. Molecular cancer(2020) 19: doi: 10.1186/s12943020-01234-1

36. Marconato L, Aresu L, Stefanello D, Comazzi S, Martini V, Ferrari R, Riondato F, Rouquet N, Frayssinet P, Sabattini S. Opportunities and challenges of active immunotherapy in dogs with B-cell lymphoma: a 5-year experience in two veterinary oncology centers. Journal for immunotherapy of cancer (2019) 7: doi: 10.1186/s40425-019-0624-y

37. Belmans J, Van Woensel M, Creyns B, Dejaegher J, Bullens DM, Van Gool SW. Immunotherapy with subcutaneous immunogenic autologous tumor lysate increases murine glioblastoma survival. Scientific reports (2017) 7: doi: 10.1038/s41598-017-12584-0

38. Hellebrekers DMEl, Castermans K, Viré E, Dings RPM, Hoebers NTH, Mayo KH, Oude Egbrink MGA, Molema G, Fuks F, van Engeland $M$, et al. Epigenetic regulation of tumor endothelial cell anergy: silencing of intercellular adhesion molecule-1 by histone modifications. Cancer research (2006) 66: doi: 10.1158/00085472.CAN-06-1609

39. van Gisbergen KPJM, Aarnoudse CA, Meijer GA, Geijtenbeek TBH, van Kooyk Y. Dendritic cells recognize tumor-specific glycosylation of carcinoembryonic antigen on colorectal cancer cells through dendritic cellspecific intercellular adhesion molecule-3-grabbing nonintegrin. Cancer research (2005) 65: doi: 10.1158/0008-5472.CAN-04-4140

40. Wang Y, Wang M, Liang H, Yu Q, Yan Z, Kong M. Imaging and clinical properties of inflammatory demyelinating pseudotumor in the spinal cord. Neural regeneration research (2013) 8: doi: 10.3969/j.issn.1673-5374.2013.26.010

41. Lau D, Garçon F, Chandra A, Lechermann LM, Aloj L, Chilvers ER, Corrie PG, Okkenhaug K, Gallagher FA. Intravital Imaging of Adoptive T-Cell Morphology, Mobility and Trafficking Following Immune Checkpoint 
Inhibition in a Mouse Melanoma Model. Frontiers in immunology (2020) 11: doi:

10.3389/fimmu.2020.01514

42. Kaufman HL, Amatruda T, Reid T, Gonzalez R, Glaspy J, Whitman E, Harrington K, Nemunaitis J, Zloza A, Wolf $\mathrm{M}$, et al. Systemic versus local responses in melanoma patients treated with talimogene laherparepvec from a multi-institutional phase II study. Journal for immunotherapy of cancer (2016) 4: doi: 10.1186/s40425-016-0116-2

43. Ye Y, Kuang X, Xie Z, Liang L, Zhang Z, Zhang Y, Ma F, Gao Q, Chang R, Lee H-H, et al. Small-molecule MMP2/MMP9 inhibitor SB-3CT modulates tumor immune surveillance by regulating PD-L1. Genome medicine (2020) 12: doi: 10.1186/s13073-020-00780-z

44. lida Y, Yoshikawa R, Murata A, Kotani H, Kazuki Y, Oshimura M, Matsuzaki Y, Harada M. Local injection of CCL19-expressing mesenchymal stem cells augments the therapeutic efficacy of anti-PD-L1 antibody by promoting infiltration of immune cells. Journal for immunotherapy of cancer (2020) 8: doi: 10.1136/jitc2020-000582

45. Cimen Bozkus C, Roudko V, Finnigan JP, Mascarenhas J, Hoffman R, lancu-Rubin C, Bhardwaj N. Immune Checkpoint Blockade Enhances Shared Neoantigen-Induced T-cell Immunity Directed against Mutated Calreticulin in Myeloproliferative Neoplasms. Cancer discovery (2019) 9: doi: 10.1158/2159-8290.CD-181356

46. Geng F, Bao X, Dong L, Guo Q-Q, Guo J, Xie Y, Zhou Y, Yu B, Wu H, Wu J-X, et al. Doxorubicin pretreatment enhances $\mathrm{FAPa}$ /survivin co-targeting DNA vaccine anti-tumor activity primarily through decreasing peripheral MDSCs in the 4T1 murine breast cancer model. Oncoimmunology (2020) 9: doi:

10.1080/2162402X.2020.1747350

47. Pirozyan MR, McGuire HM, Emran AA, Tseng H-Y, Tiffen JC, Lee JH, Carlino MS, Menzies AM, Long GV, Scolyer RA, et al. Pretreatment Innate Cell Populations and CD4 T Cells in Blood Are Associated With Response to Immune Checkpoint Blockade in Melanoma Patients. Frontiers in immunology (2020) 11: doi: 10.3389/fimmu.2020.00372

48. Sanner T, Grimsrud TK. Nicotine: Carcinogenicity and Effects on Response to Cancer Treatment - A Review. Front Oncol(2015) 5:196-196. doi: 10.3389/fonc.2015.00196

49. Schaal C, Chellappan SP. Nicotine-mediated cell proliferation and tumor progression in smoking-related cancers. Molecular cancer research: MCR (2014) 12: doi: 10.1158/1541-7786.MCR-13-0541

50. Grando SA. Connections of nicotine to cancer. Nature reviews Cancer(2014) 14: doi: 10.1038/nrc3725

\section{Tables}

Table 1. Cox proportional hazards regression model analysis of overall survival in TCGA. 


\begin{tabular}{|c|c|c|c|c|c|c|c|c|}
\hline & $\mathrm{HR}$ & HR.95L & HR.95H & $\begin{array}{l}P \\
\text { value }\end{array}$ & $\mathrm{HR}$ & HR.95L & HR.95H & $\begin{array}{l}p \\
\text { value }\end{array}$ \\
\hline Grade(II, III and IV) & 4.485 & 3.431 & 5.863 & $<0.001$ & 1.827 & 1.348 & 2.476 & $<0.001$ \\
\hline IDH.status(Yes vs NO) & 0.106 & 0.075 & 0.150 & $<0.001$ & 0.339 & 0.195 & 0.590 & $<0.001$ \\
\hline $\begin{array}{l}\text { Chr1p19q.codeletion(Yes } \\
\text { vs No) }\end{array}$ & 0.232 & 0.138 & 0.391 & $<0.001$ & 0.611 & 0.321 & 1.160 & 0.132 \\
\hline Age & 1.061 & 1.048 & 1.074 & $<0.001$ & 1.037 & 1.022 & 1.052 & $<0.001$ \\
\hline Gender(Male vs Female) & 1.175 & 0.845 & 1.632 & 0.338 & 1.181 & 0.832 & 1.675 & 0.352 \\
\hline ITGAL & 1.366 & 1.220 & 1.531 & $<0.001$ & 1.151 & 1.001 & 1.323 & 0.048 \\
\hline
\end{tabular}

Table 2. Cox proportional hazards regression model analysis in CGGA.

\begin{tabular}{|c|c|c|c|c|c|c|c|c|}
\hline \multirow[t]{2}{*}{ Variables } & \multicolumn{4}{|c|}{ Univariate analysis } & \multicolumn{4}{|c|}{ Multivariate analysis } \\
\hline & HR & HR.95L & HR.95H & $\begin{array}{l}P \\
\text { value }\end{array}$ & HR & HR.95L & HR.95H & $\begin{array}{l}P \\
\text { value }\end{array}$ \\
\hline Grade(II, III and IV) & 2.857 & 2.491 & 3.277 & $<0.001$ & 2.062 & 1.755 & 2.423 & $<0.001$ \\
\hline IDH.status(Yes vs NO) & 0.305 & 0.250 & 0.372 & $<0.001$ & 0.616 & 0.483 & 0.785 & $<0.001$ \\
\hline $\begin{array}{l}\text { Chr1p19q.codeletion(Yes } \\
\text { vs No) }\end{array}$ & 0.247 & 0.182 & 0.333 & $<0.001$ & 0.436 & 0.313 & 0.607 & $<0.001$ \\
\hline Radio status(Yes vs No) & 1.148 & 0.860 & 1.532 & 0.349 & 0.834 & 0.611 & 1.140 & 0.255 \\
\hline $\begin{array}{l}\text { Chemo status(Yes vs } \\
\text { No) }\end{array}$ & 1.937 & 1.535 & 2.445 & $<0.001$ & 0.877 & 0.675 & 1.141 & 0.330 \\
\hline Age & 1.032 & 1.023 & 1.042 & $<0.001$ & 1.011 & 1.002 & 1.020 & 0.021 \\
\hline Gender(Male vs Female) & 1.029 & 0.846 & 1.252 & 0.774 & 1.121 & 0.917 & 1.369 & 0.264 \\
\hline ITGAL & 1.407 & 1.286 & 1.540 & $<0.001$ & 1.135 & 1.024 & 1.258 & 0.016 \\
\hline
\end{tabular}

Table 3. Baseline Patient Characteristics of Included Observations from TCGA and CGGA. 


\begin{tabular}{|c|c|c|}
\hline Variables & TCGA Cohort $(n=603)$ & CGGA Cohort $(n=681)$ \\
\hline \multicolumn{3}{|l|}{ Gender } \\
\hline female & $254(42.1)$ & $285(41.9)$ \\
\hline male & 349 (57.9) & $396(58.1)$ \\
\hline \multicolumn{3}{|l|}{ Age group } \\
\hline$<=45$ & $289(47.9)$ & 417 (61.2) \\
\hline$>45$ & $314(52.1)$ & $264(38.8)$ \\
\hline \multicolumn{3}{|l|}{ Grade } \\
\hline WHO II & $213(35.3)$ & $213(31.3)$ \\
\hline WHO III & $238(39.5)$ & $230(33.8)$ \\
\hline WHO IV & $152(25.2)$ & $238(34.9)$ \\
\hline \multicolumn{3}{|l|}{ Histology } \\
\hline LGG & $451(74.8)$ & $443(65.1)$ \\
\hline GBM & $152(25.2)$ & $238(34.9)$ \\
\hline \multicolumn{3}{|c|}{ IDH1 mutation status } \\
\hline wild type & $224(37.1)$ & $283(41.6)$ \\
\hline mutant type & 373 (61.9) & $398(58.4)$ \\
\hline unkown & $6(1.00)$ & \\
\hline \multicolumn{3}{|c|}{ 1p19q co-deletion status } \\
\hline codel & $149(24.7)$ & $521(76.5)$ \\
\hline non-codel & $449(74.5)$ & $160(23.5)$ \\
\hline unkown & $5(00.8)$ & \\
\hline \multicolumn{3}{|c|}{ Chemotherapy } \\
\hline yes & unkown & $477(70.0)$ \\
\hline no & unkown & $204(30.0)$ \\
\hline \multicolumn{3}{|l|}{ Radiotherapy } \\
\hline yes & unkown & 577 (84.7) \\
\hline no & unkown & $104(15.3)$ \\
\hline
\end{tabular}

\section{Figures}


A
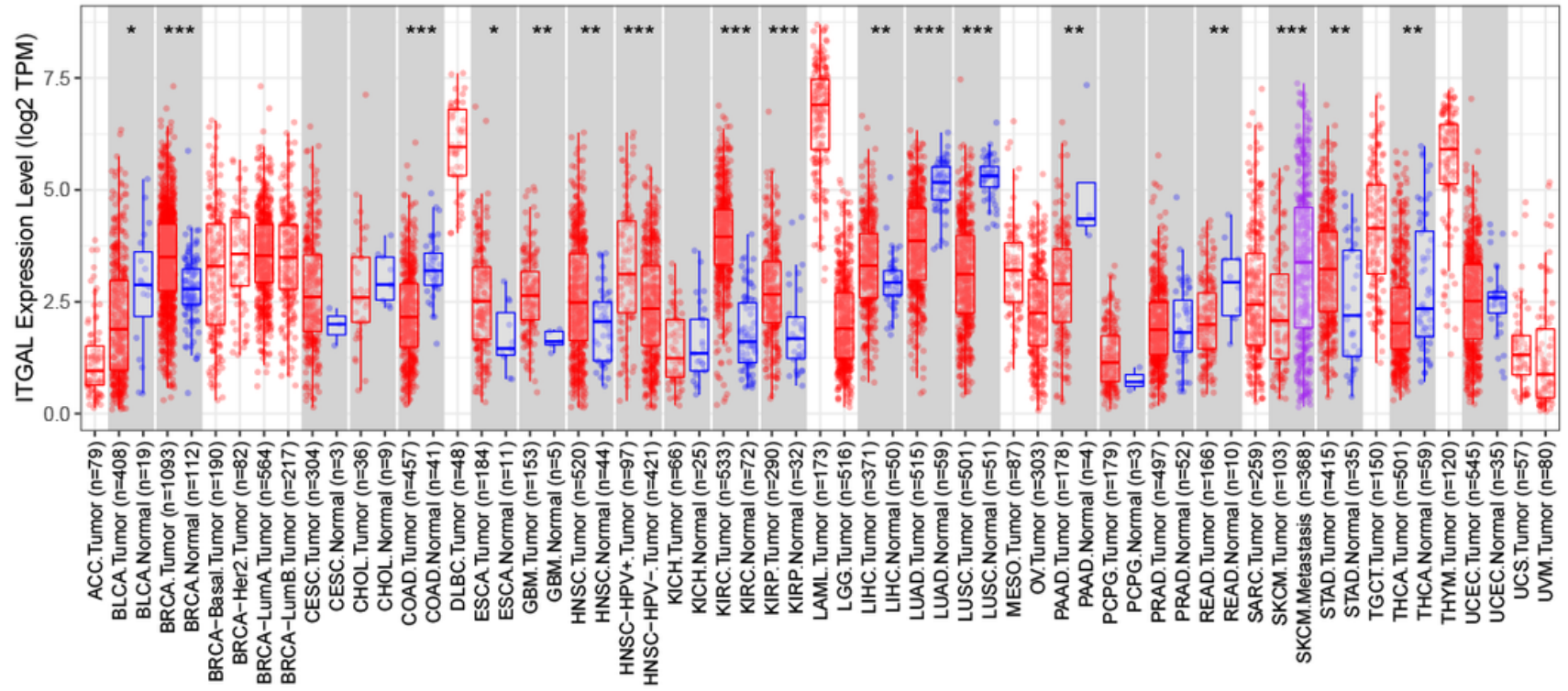

B
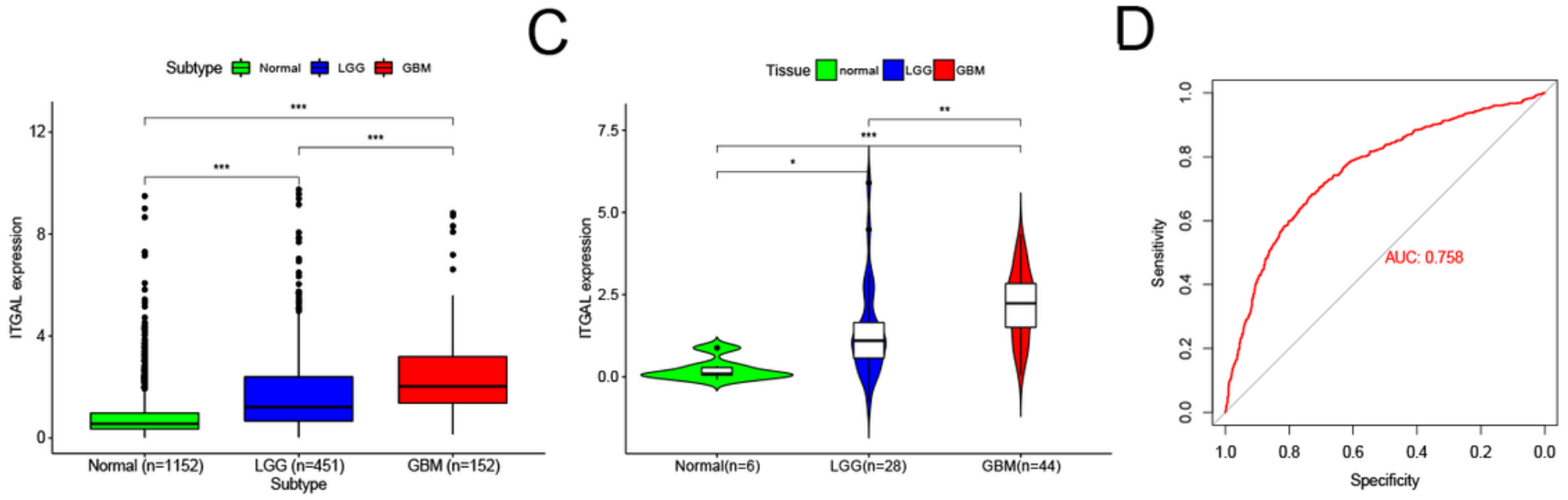

Figure 1

ITGAL expression level in human tissues and the evaluation of its diagnostic value in glioma. (A) ITGAL mRNA expression in different normal human tissues and cancer tissues. (B) Comparison of ITGAL mRNA expression in normal tissues and cancer tissues in LGG and GBM in training set. (C) The level of ITGAL in normal, LGG and GBM tissues in our hospital samples. LGG: low grade glioma, GBM: glioblastoma. (D) ROC curve analysis revealed that the overexpression of ITGAL had a high sensitivity and specificity to diagnose glioma, AUC $=0.758$. (ns: no significance, ${ }^{\star} \mathrm{P}<0.05,{ }^{\star} \mathrm{P}<0.01, \star \star \star \mathrm{P}<0.001$ ). 

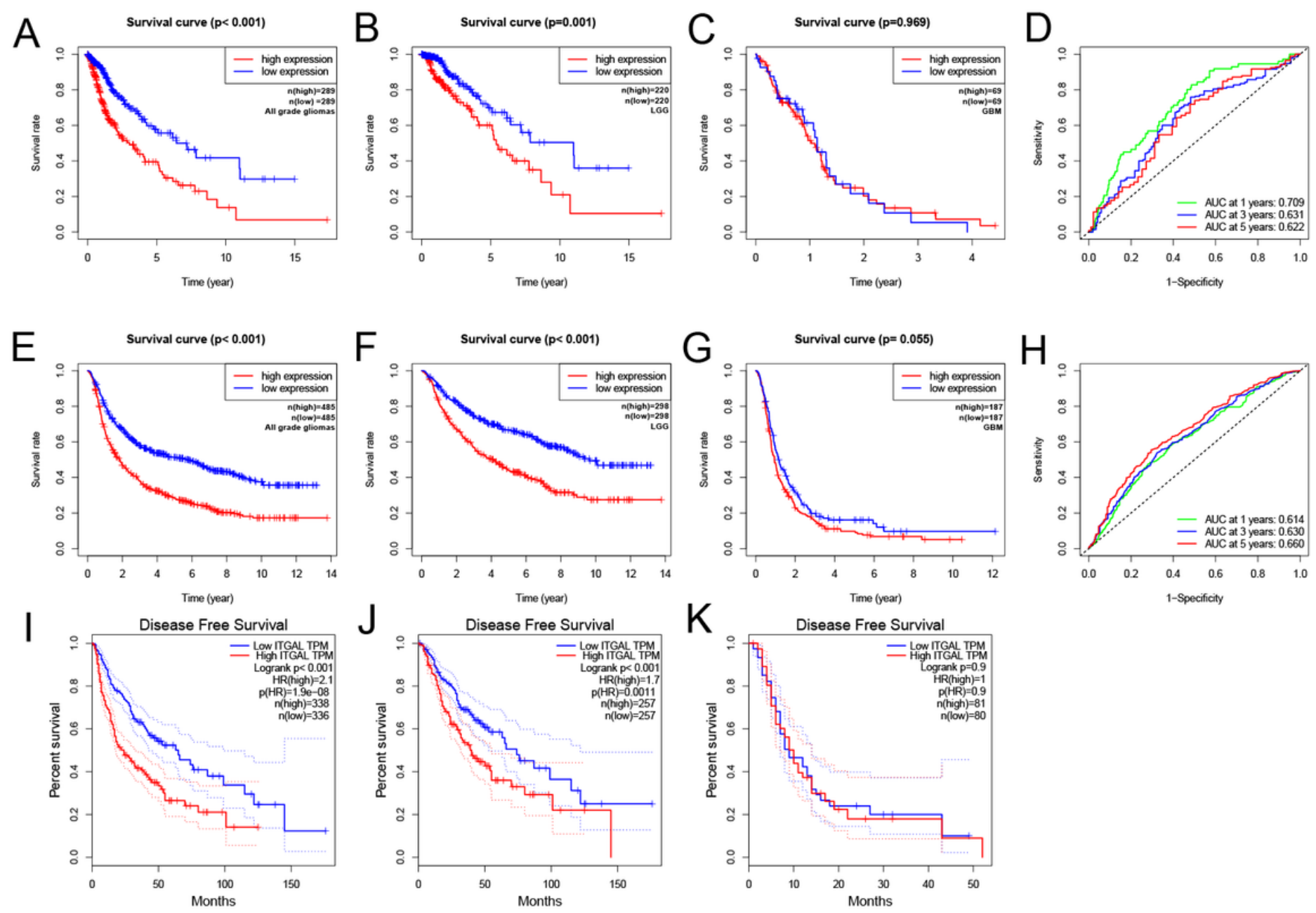

\section{Figure 2}

The prognostic value of ITGAL in glioma. According to the median value of ITGAL, patients were divided into low and high expression group. In TCGA-glioma cohort, K-M curves performed to investigate the correlation between ITGAL and OS in (A) all grade gliomas, (B) LGG and (C) GBM. (D) The AUC of ROC for predicting the 1-, 3- and 5-year OS of patients in TCGA was $0.709,0.631$ and 0.622 respectively. In CGGA cohort, K-M curves performed in (E) all grade glioma, (F)LGG and (G) GBM. (H) The AUC of ROC for predicting the 1-, 3- and 5-year OS of patients in CGGA was $0.614,0.630$ and 0.660 respectively. According to the GEPIA database, K-M survival analysis performed to explore the correlation between ITGAL and DFS in (I) all grade glioma, (J) LGG and (K) GBM. OS: overall survival, LGG: lower grade glioma, GBM: glioblastoma, DFS: disease free survival. 

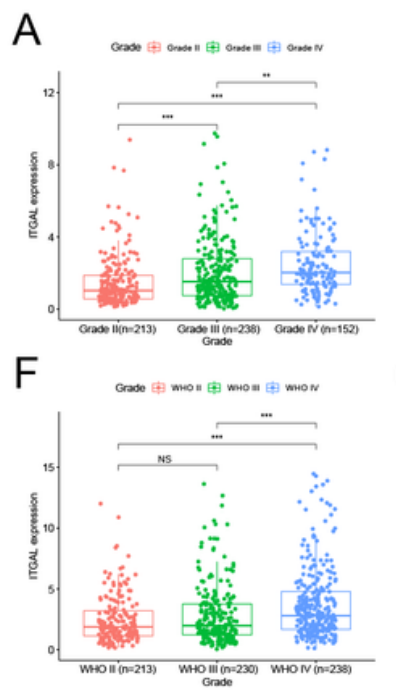

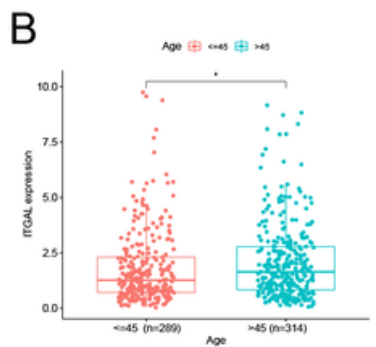

G $\quad H$



C

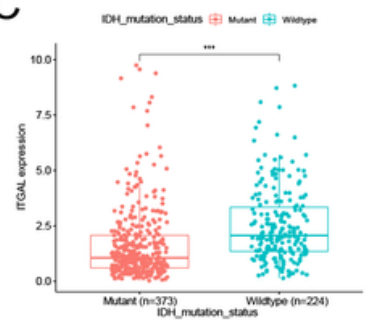

$\mathrm{H}$

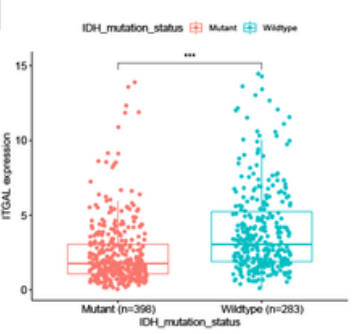

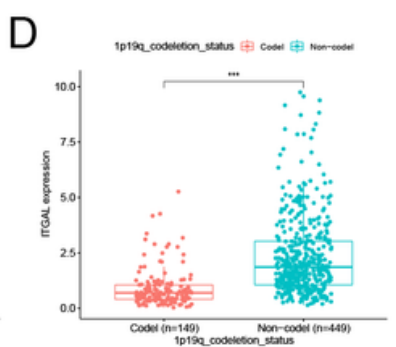
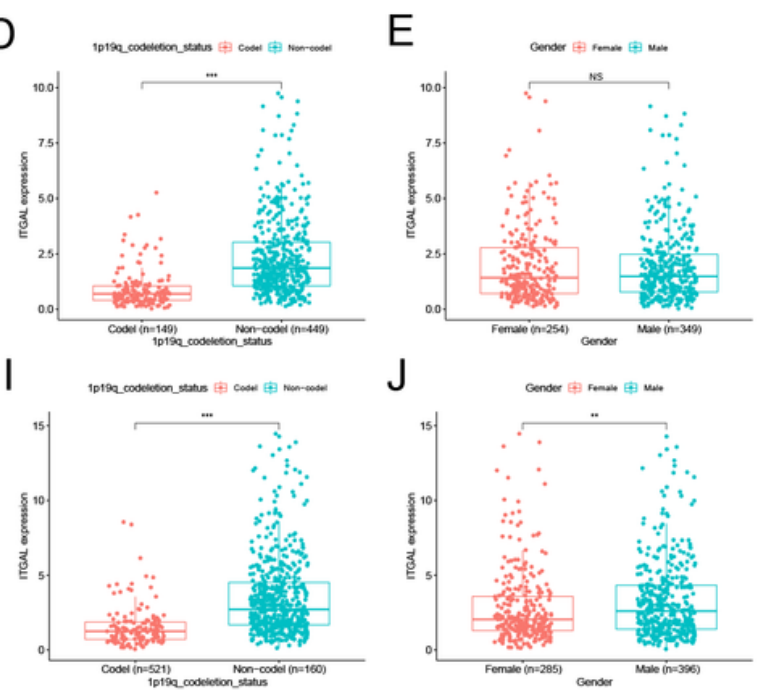

Figure 3

The association between ITGAL and clinicopathological characteristics. In TCGA cohort, ITGAL expression levels were investigated in different (A) WHO grade, (B) age groups, (C) IDH status, (D) 1p19q co-deletion state and $(E)$ gender. In CGGA cohort, the expression levels of ITGAL were investigated in different (F) WHO grade, $(G)$ age group, (H) IDH status, (I) 1p19q co-deletion and (J) gender. ***: $p<0.001, * *: p<0.01, *: p<0.05$, NS: not significant.
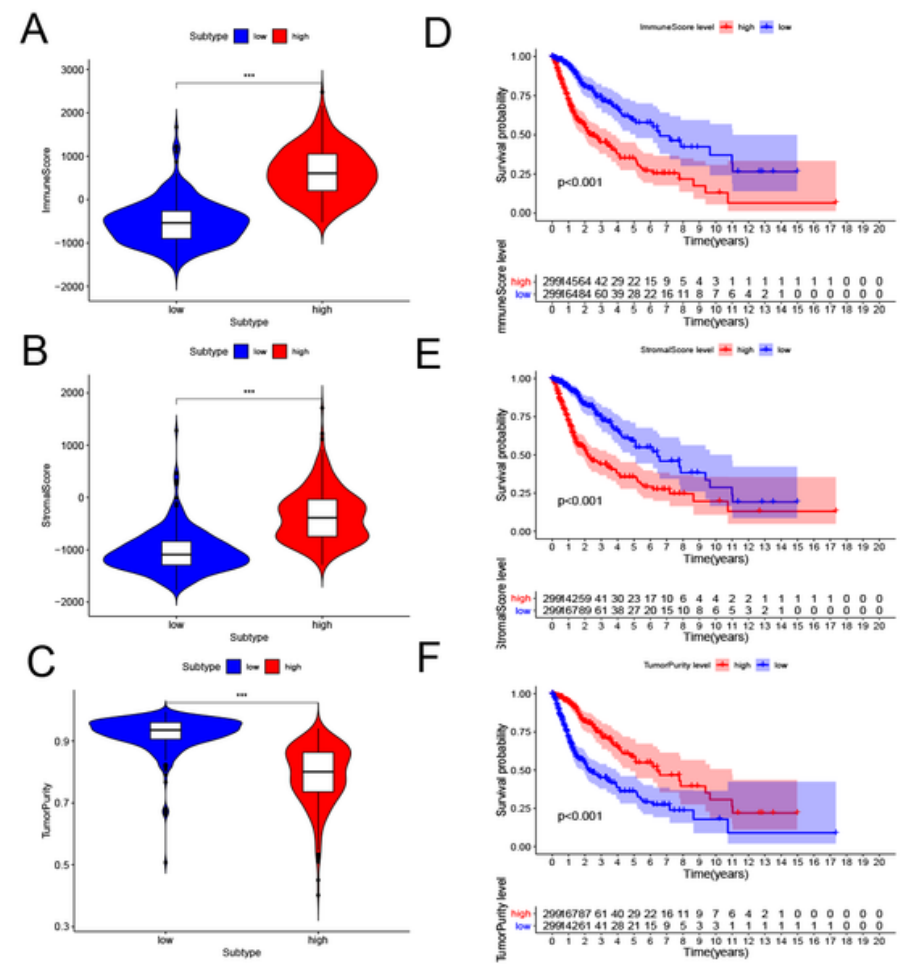

E
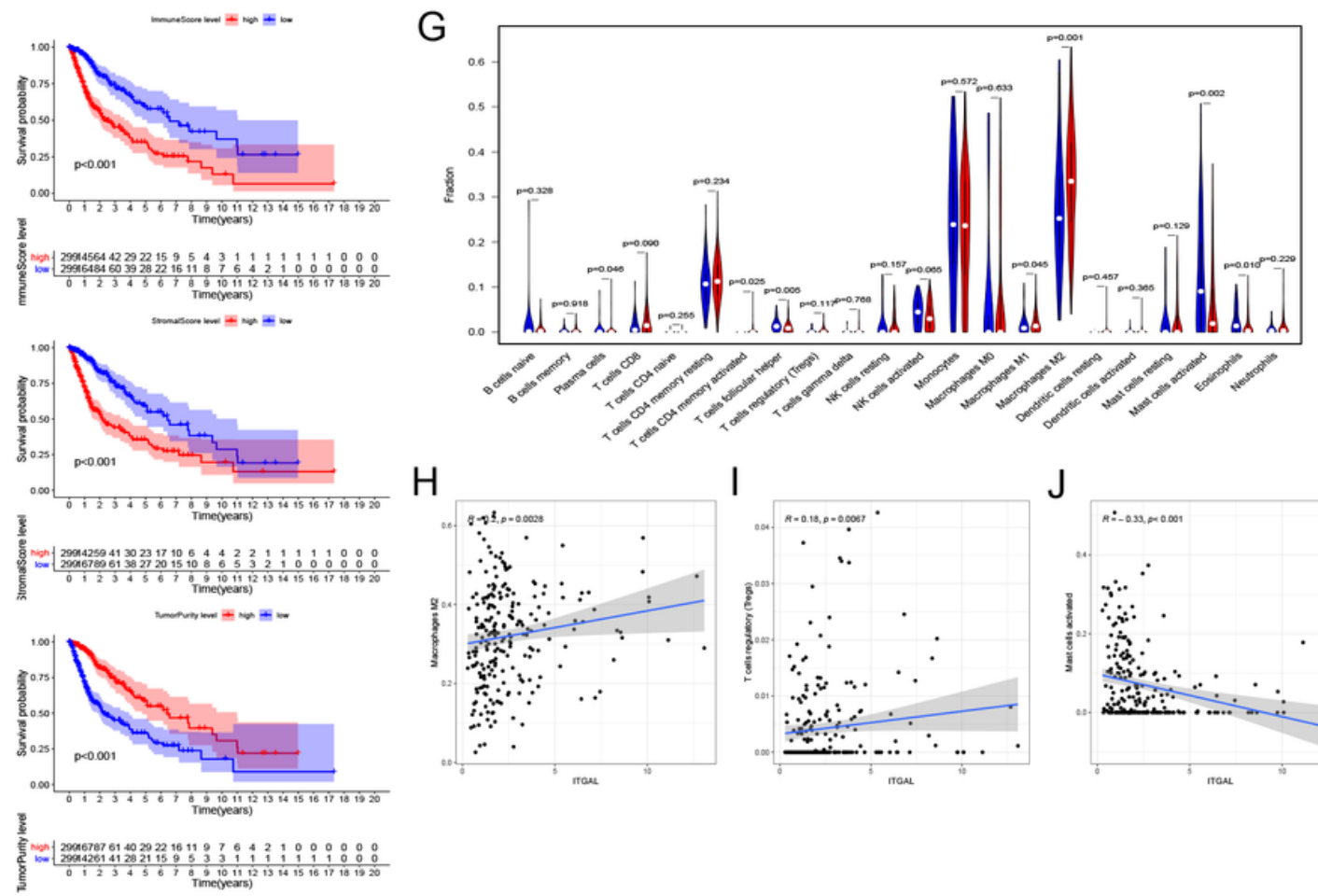

$\mathrm{H}$

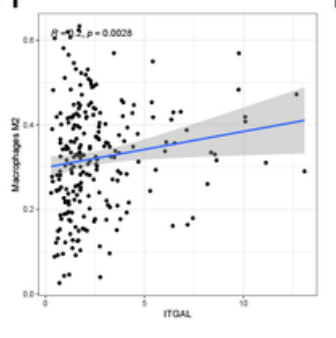

$\mathrm{J}$

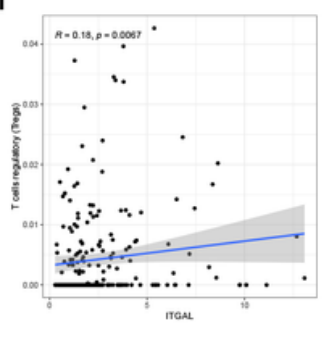

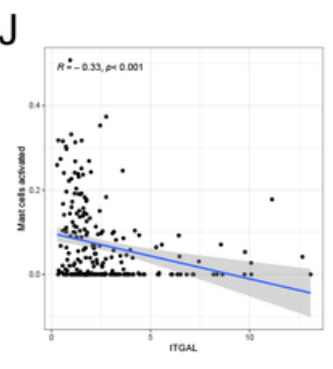

\section{Figure 4}

The correlation between immunity and ITGAL in TCGA-glioma. 
Patients were divided into low and high expression by the median value of ITGAL. The value of (A) immune score, (B)stromal score and (C) tumor purity in low and high expression group. K-M performed to investigate the relationship between OS and (D) immune score, (E) stromal score and (F) tumor purity. (G) The fraction level of tumor infiltrating immune cells (TIICs) in the high and low expression group of ITGAL in glioma in TCGA. The correlation analysis between ITGAL and (H) M2 macrophages, (I) regulatory T cells and $(\mathrm{J})$ actived mast cells. $* * *: p<0.001$.

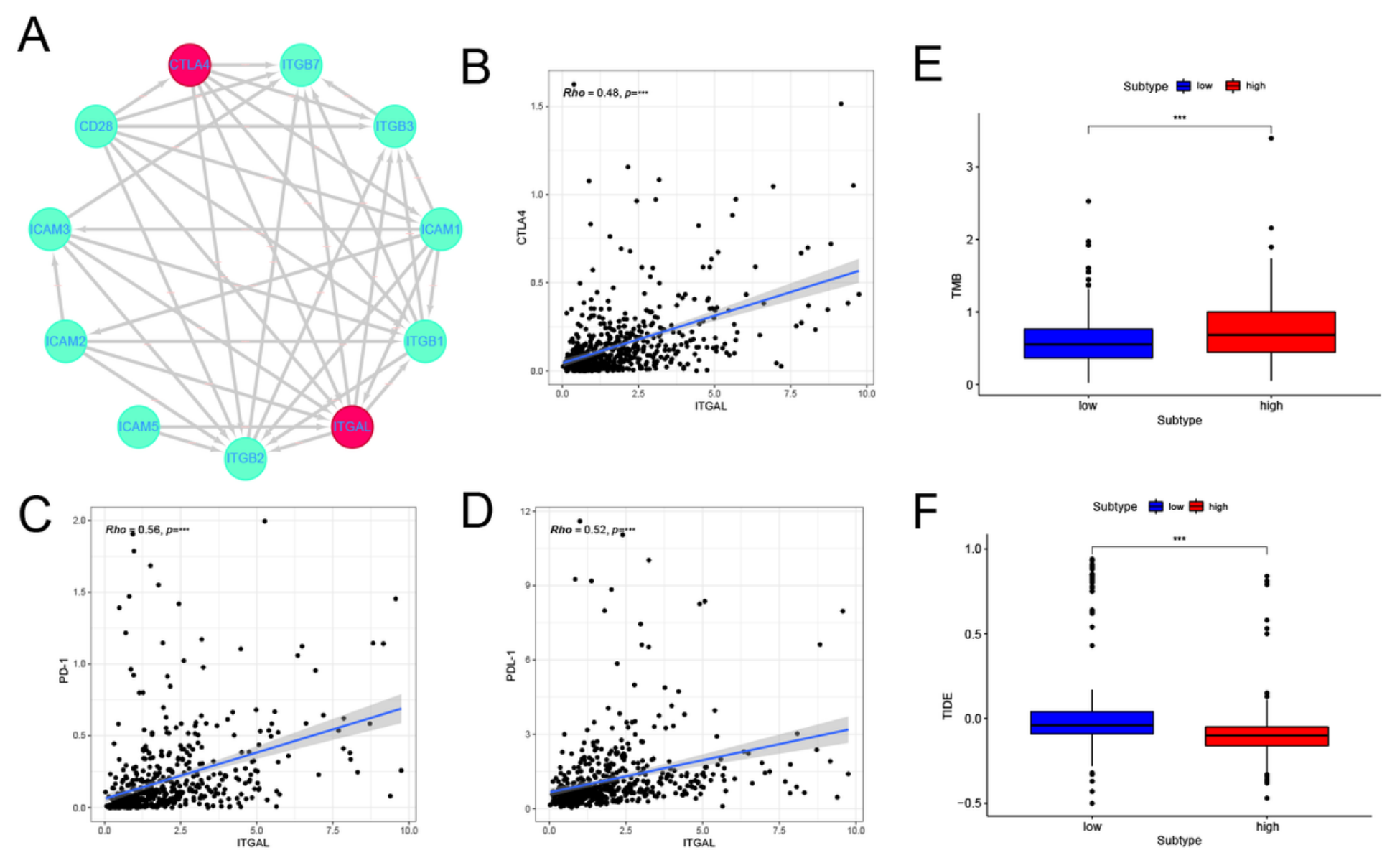

Figure 5

ITGAL could be used for predicting immunotherapeutic benefit. (A) PPI networks of ITGAL in STRING database re-built by Cytoscape.The solid one-sided arrows represent up- or down-regulations. The expression of ITGAL was positively correlated with (B) CTLA-4, (C) PD-1 and (D) PDL-1 according to the spearman correlation analysis. (E) The value of TMB in low and high ITGAL expression group. (F) Score of TIDE in low and high expression group. TMB: tumor mutation burden, TIDE: Tumor Immune Dysfunction and Exclusion, $\star \star \star: ~ p<0.001$. 


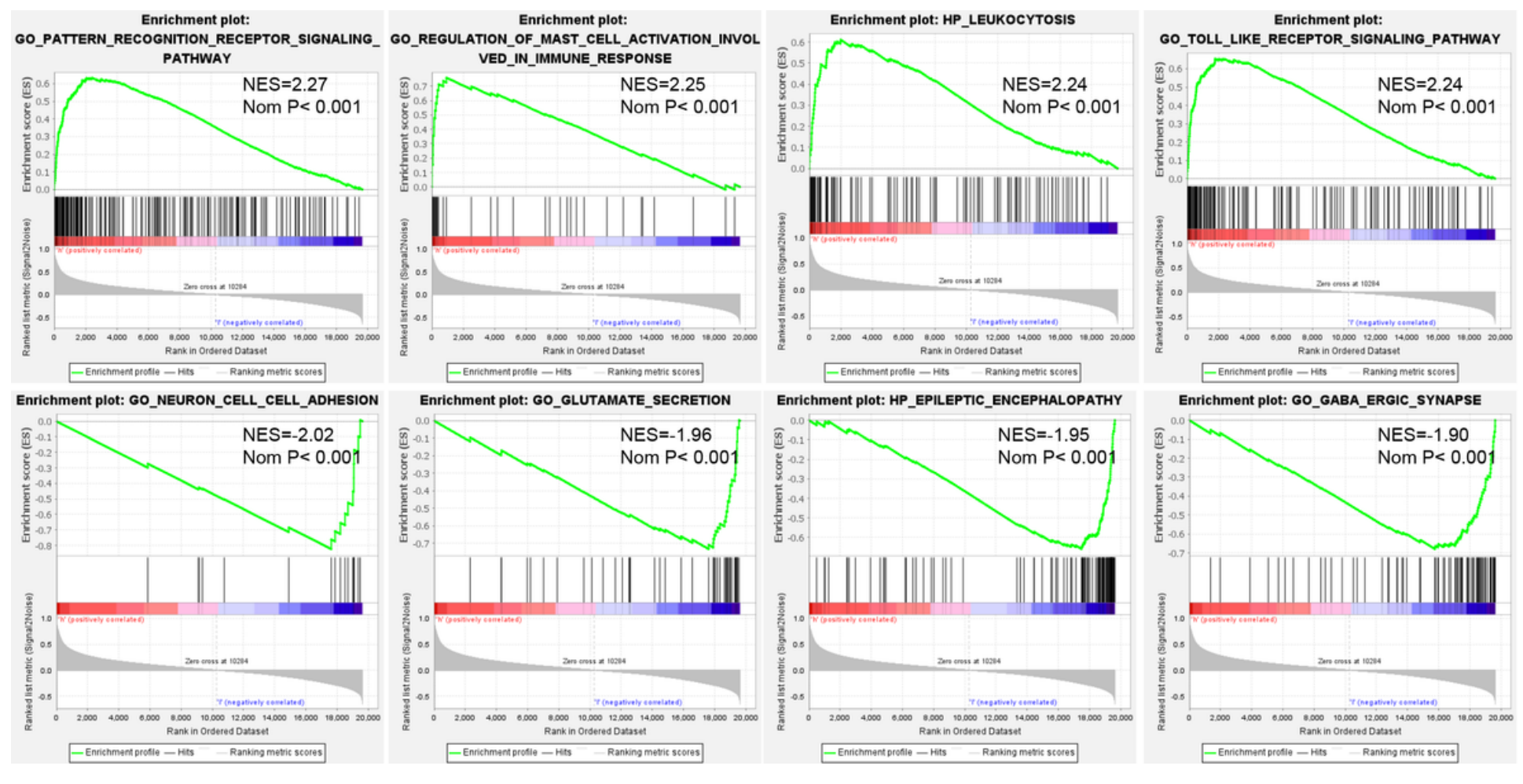

Figure 6

Gene sets enrichment analysis of ITGAL in TCGA-glioma. The top three enriched terms positively and negatively related to the value of ITGAL were shown. NES: normalized enrichment score, Nom P: nominal $p$ value, h: high expression group of ITGAL, I: low expression group of ITGAL.

A

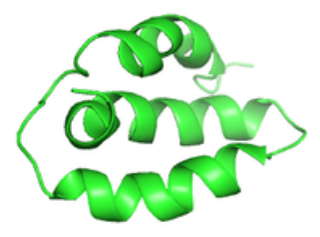

C

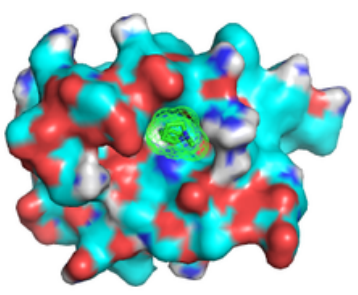

B

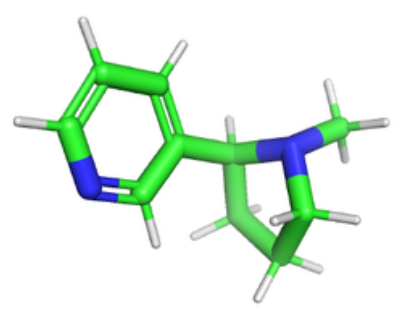

D

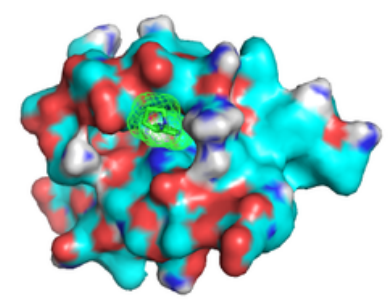

E

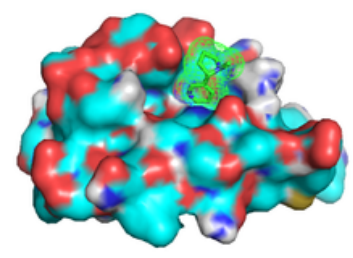

Figure 7

Ingredient in related traditional Chinese medicine targeting ITGAL protein. (A) Secondary structure of ITGAL protein. (B) 3D structure of nicotine. (C-E) Molecular docking among ITGAL protein and nicotine was viewed from different angles. 